\title{
Talimogene Laherparepvec Regimen
}

National Cancer Institute

\section{Source}

National Cancer Institute. Talimogene Laherparepvec Regimen. NCI Thesaurus. Code C160814.

A regimen consisting of intralesional talimogene laherparepvec that may be used in the treatment of recurrent, unresectable, cutaneous, subcutaneous, and nodal melanoma lesions. 